\title{
La escuela jurídica kantiana y la ciencia del derecho en Alemania (1750-1804)
}

The Kantian legal school and the science of law in Germany (1750-1804)

\author{
Jesús Miguel Santos Román ${ }^{1}$ \\ Universidad Francisco de Vitoria
}

Recibido: 15.12 .2020

Aceptado: 29.12.2020

\section{Resumen}

Texto correspondiente a la defensa de la Tesis Doctoral del autor, que lleva por título La escuela jurídica kantiana y la ciencia del derecho en Alemania (1750-1804), en la que se examina el desenvolvimiento coherente del iusnaturalismo inmanentista alemán, a lo largo del periodo ilustrado, en su apelación a la ciencia jurídica (Rechtslehre), por medio de los conceptos a priori de naturaleza e historia.

Palabras clave: Kant, escuela kantiana, ciencia jurídica, derecho natural, Naturrecht.

\begin{abstract}
Text corresponding to the public $\mathrm{PhD}$ dissertation entitled The Kantian Legal School and the Science of Law in Germany (1750-1804) by the author, which examines the consistent development of immanentistic German Jusnaturalism throughout the Enlightenment period
\end{abstract}

1jesusmiguel.santos@ufv.es

https://orcid.org/0000-0002-2534-7663

Volumen 1. Número 2. Julio - Diciembre 2021

61 ISSN: 2745-0333 (En línea) 
regarding its appeal to the Science of Law (Rechtslehre) by means of the a priori concepts of nature and history.

Key words: Kant, kantian school, science of law, natural law, naturrecht.

\section{La investigación}

El núcleo central de mi investigación comenzaba a gestarse en las sesiones del Seminario Permanente de Filosofía del Derecho, que daba sus primeros pasos en esta misma Facultad de Derecho allá por el año 2008, bajo la dirección del Prof. Evaristo Palomar.

Movido por el deseo de entender la ciencia jurídica tal cual la había recibido de mis profesores $\mathrm{y}$, en general, la forma mentis que anima al jurista contemporáneo, se fue colocando en el centro de mi atención, gracias a la ayuda de mi Director, el problema del concepto de naturaleza.

Este leitmotiv nos hizo volver la mirada hacia las raíces de quien es ampliamente reconocido como un hito insoslayable en la historia del pensamiento jurídico: Kant. Sin embargo, la lectura sostenida de la obra del Prof. Dr. Francisco Carpintero Benítez, Catedrático Emérito de la Facultad de Derecho de la Universidad de Cádiz, me suscitó el interés por comprobar directamente desde las fuentes si la imagen que había recibido del filósofo de Königsberg, y la profunda influencia de su pensamiento en los desarrollos doctrinales posteriores, era todo lo rigurosa que cabría desear.

Se puede decir que, en España, la recepción de Kant ha sido tardía, en un primer momento indirecta y, si se mira desde el punto de vista de la doctrina kantiana en general, incompleta. Como he tenido ocasión de reflejar al comienzo de la investigación, a una primera lectura indirecta desde las traducciones francesas, a lo largo del siglo XIX siguió, a finales de ese siglo, una recepción a través del prurito de ciencia infundido por la influencia de la escuela histórica de derecho en suelo patrio. Poco a poco, con la llegada del siglo XX, el interés 
académico creciente por la cultura germana, y el trabajo de diversos compatriotas becados para culminar sus estudios en Alemania, fue incrementando el interés por la lectura de Kant, pero ya desde posiciones neokantianas, idealistas, o incluso marxistas. Ruego se me excuse de una exposición detallada al respecto. La recepción de Kant en España se me presenta como una indagación con autonomía propia, que tengo intención prolongar entre mis líneas de investigación futuras.

\subsection{Naturaleza}

Mi Tesis Doctoral es una investigación en filosofía del derecho. Cierto que no es ajena al desenvolvimiento histórico de los desarrollos científicos del periodo, pero en ningún caso pretende presentarse como un estudio en historia de la filosofía, ni su objeto se ciñe a establecer la secuencia de los acontecimientos académicos más relevantes en orden cronológico. El propósito de la investigación consiste en el estudio directo y contextualizado de las fuentes doctrinales, en un marco espaciotemporal al que frecuentemente se apela por haber contribuido con un impulso definitivo a la necesidad de hallar las condiciones racionales por las que el derecho podía y debía llegar a convertirse en ciencia.

\subsection{Delimitación}

Así pues, la investigación quedaba delimitada al contexto académico germano, dentro del periodo que va desde 1750 a 1804. Toda demarcación histórica, en cuanto toma de posición metódica, puede resultar discutible desde diversos puntos de vista. Me corresponde, por tanto, exponer mis razones para escoger una y otra fecha.

Con esta delimitación se pretendía abarcar el apogeo de la Ilustración alemana (die Aufklärung). Como se puede observar en el cuerpo de la investigación, la década de los cincuenta del siglo XVIII supone un cambio de frente en el panorama académico y cultural germano. El retorno de Wolff a Halle, el ascenso de Federico el Grande al trono de Prusia y 
la reforma de las Universidades suponen, entre otros elementos, el fermento adecuado para una rápida expansión de la Naturrechtsphilosophie a lo largo y ancho de la geografía alemana. Sin este contexto, tampoco se entendería el triunfo apoteósico del kantismo, que, en el ámbito jurídico, extiende su influencia desde la década de los noventa del siglo XVIII hasta prácticamente los años treinta del siglo XIX. Aun así, como se recoge en el título de mi Tesis Doctoral, el año de 1804 marca el final de la investigación. El fallecimiento de Kant coincide con el punto álgido de la escuela fundada por él. A partir de ese momento, se generaliza el viraje de muchos autores hacia posiciones metódicas y doctrinales más novedosas, y en auge, como el idealismo, o la escuela histórica del derecho, de manera que las obras de quienes permanecen bajo el signo kantiano pierden originalidad y casi constituyen una repetición de los tópicos bien conocidos del periodo anterior. Asimismo, la abolición del Imperio Romano por Napoleón en 1806, alumbraba un mundo nuevo con características propias, cuyo estudio pospongo para futuras investigaciones.

\section{Método}

El método utilizado es conceptual y exegético, compuesto por una primera fase analítica, y una segunda de naturaleza sintética. Previamente, la selección de los autores y las obras más representativas del periodo, salvo las del propio Kant, siguió el criterio de optar por aquellas fuentes que estuvieran directamente vinculadas al ámbito jurídico, y solo excepcionalmente otras obras de carácter moral, siempre que al menos abordaran el concepto de derecho, aunque fuera de forma indirecta.

En esta labor delimitativa, fue de inestimable ayuda la producción científica del Dr. Carpintero Benítez. Asimismo, he de remarcar la trascendencia de obras como la de Warnkönig, en su tratado Rechtsphilosophie als Naturlehre des Rechts (1839), Liebmann y su Kant und die Epigonen (1865) y Blüdhorn con su artículo „,Kantianer“ und Kant. Die Wende von der Rechtsmetaphysik zur ,Wissenschaft“ vom positiven Recht (1973). Por supuesto, son incontables las referencias cruzadas que he encontrado en las obras de los propios autores del periodo. 
Una vez seleccionadas las fuentes, o de forma concomitante, comenzó la fase analítica, en una búsqueda y selección de los conceptos nucleares que pudieran ofrecerme las claves interpretativa del desarrollo doctrinal del periodo.

La fase más trabajosa fue, sin duda, la búsqueda de una síntesis ordenada al entender, y a conseguir una exposición cabal de la columna vertebral que recorre el quehacer filosófico de aquellos pensadores. Mediante una labor comparativa, y un ir y venir desde las consecuencias a los principios, y desde los principios a las consecuencias, fui puliendo el despliegue argumental que aparece recogido en el texto de mi investigación.

\subsection{Precisión terminológica}

Como se puede observar, he seguido la terminología utilizada por los académicos alemanes contemporáneos a la hora de distinguir dos corrientes doctrinales protagonistas a lo largo del periodo objeto de estudio.

De un lado estaría el llamado älteres Naturrecht, el derecho natural antiguo o, según un neologismo de mi invención, el veteroiusnaturalismo. Esta corriente abarcaría desde los orígenes del iusnaturalismo en la Modernidad hasta la entrada en escena del kantismo, de manera que no es una categoría exclusiva del marco temporal de mi investigación. Ciñéndome exclusivamente a los límites de dicho marco temporal, esta primera etapa estará signada por el éxito del wolffianismo que, por temática y por estilo, una buena parte de la doctrina científica alemana entronca con aquel método inmediatamente anterior, considerándolo, por tanto, como integrante del iusnaturalismo more antiquo.

En contraste con él, las fuentes secundarias consultadas encuentran que existen elementos suficientes para considerar que, con la llegada del kantismo, se produce un cambio de frente en el iusnaturalismo, de manera que se puede hablar de un jüngeres Naturrecht, un derecho natural nuevo o, según mi traducción, novoiusnaturalismo. Así pues, este novoiusnaturalismo prácticamente se identifica, con alguna salvedad, con la filosofía criticista. 
Como se trataba de atender al contexto doctrinal del kantismo, enjuiciando su originalidad o dependencia de lo anterior, a lo largo de la investigación se puede observar un ejercicio constante de comparativa entre una tendencia y otra, a fin de trazar líneas comunes o divergentes entre el veteroiusnaturalismo, de corte wolffiano y el novoiusnaturalismo, protagonizado por la filosofía criticista. Hasta aquí la justificación, el objeto y el método de mi Tesis Doctoral.

Mi estudio se acompaña de dos anexos, con la transcripción y traducción de las obras de Ludwig Julius Friedrich Höphner, Naturrecht des einzelen Menschen, der Gesellschaften und der Völker, de 1780, y de Wilhelm Traugott Krug, Aphorismen zur Philosophie des Rechts, de 1800, por lo que ambas tienen de representativo, tanto del vetero como del novoiusnaturalismo, respectivamente.

\subsection{Hipótesis}

De todos es conocido que la lectura de Hume había de producir en Kant el celebérrimo despertar del sueño dogmático (das Erwachen aus dem dogmatischen Schlaf), que, según se dice, lo apartaría de su pasado racionalista. La necesidad de superar la aparente dicotomía entre racionalismo y empirismo impulsaría al filósofo de Königsberg, a lo largo de un holgado periodo de silencio intelectual, a erigir un edificio doctrinal que sorteara a toda costa la falacia naturalista denunciada por Hume.

La filosofía criticista, cuya eclosión coincide con la publicación de la primera edición de la Kritik der reinen Vernunft en 1781, se postulaba como árbitro entre dos reinos contendientes: de un lado, el dominio de un uso teorético de la razón, de otro, el dominio de un uso práctico de la misma. De modo que, delimitados los pagos de cada cual, ni el sistema de la naturaleza volviera jamás a sentirse tentado de inmiscuirse en el horizonte del espíritu -lo que, sin duda, supondría disolver todo enunciado moral en la multiplicidad caótica del torrente empírico (escepticismo empirista)-, ni tampoco el sistema del espíritu volviera a sentirse tentado de 
inmiscuirse en los asuntos del horizonte fenoménico -cosa que solo podía traer como resultado la corrupción de la ciencia física en superstición-.

Se entiende, pues, la insistencia de Kant en negar la posibilidad de toda intuición intelectual, marcando, con ello, los límites del conocimiento finito. Y este punto, ciertamente, lo distancia del racionalismo anterior, como ha sido ampliamente destacado por los estudiosos del filósofo de Königsberg.

En efecto, desde la metafísica esencialista del wolffianismo, anterior a la filosofía criticista, el existir había de revelarse como mera afección modal de la esencia, de manera que la objetualidad de lo conocido se resolvía en su esencia, esta, en la mera posibilidad, y esta última, a su vez, en la ausencia de contradicción, según aquel esquema en que el principio de razón suficiente se subsumía como un apartado del principio de no contradicción. Y así, la búsqueda de las condiciones racionales por las que la moral (y el derecho) podía y debía llegar a convertirse en una doctrina scientifica methodo pertractata, lejos de dirigirse hacia el acto de la praxis humana, había de identificarse como la derivación de un sistema de conexiones lógicas necesarias, bajo el nombre de leyes, cuyo presupuesto primero lo era la naturaleza, de donde la apelación al ius naturae. De manera que las dificultades que podía plantear una contraposición entre naturaleza y libertad, y que destaco en el apartado 2.2.1 de la investigación, en el marco del veteroiusnaturalismo wolffiano quedaban resueltas en un monismo de fondo, tendente a la contemplación de los atributos de un dios -flatus vocis-, a partir del cual se hace posible la derivación de las res in se bonae, es decir, las leyes necesarias para una praxis universal, esto es, lo que he denominado la pretendida fundamentación objetiva de la doctrina ética en el apartado 2.2.2 de la investigación.

A pesar de las diferencias ciertamente existentes entre la filosofía wolffiana y la filosofía criticista, lo cierto es que aquel prurito de ciencia fue heredado íntegramente por el novoiusnaturalismo. Pero lo que más suele sorprender a quien se aproxima al estudio de estas fuentes es el descubrimiento de que la escuela kantiana mantuviera la apelación al Naturrecht, y la summa divisio de esta disciplina jurídica en términos muy parecidos al periodo anterior. 
Porque desde la filosofía criticista, lo propio habría sido reservar el término naturaleza y sus derivados para el horizonte de un uso teorético de la razón, es decir, el horizonte del fenómeno, en su intuición empírica. Sin embargo, presupuesta la improporción de la cosa en sí, más allá de los límites del horizonte físico, las representaciones de la razón no pueden arrojar conocimiento teorético, y solo pueden revestir, por tanto, un carácter regulativo, es decir, no natural. ¿Cómo hablar, pues, de derecho natural?

Mi hipótesis inicial partía de un hecho histórico innegable: como destacará el Dr. Carpintero Benítez, la Metaphysik der Sitten es una obra tardía, y para cuando la primera parte de esta publicación, los Metaphysische Anfangsgründe der Rechtslehre viera la luz hacia finales de 1796, ya existía una extensa bibliografía con sello kantiano, en la que diversos académicos repartidos por la geografía alemana habían hecho su propio intento de erigir una doctrina jurídica desde los presupuestos metafísicos dados a conocer por el filósofo de Königsberg al comienzo de su periodo criticista. De donde se comprende la conclusión del Prof. Carpintero Benítez: Kant fue un kantiano más, y la escuela kantiana, tan solo el otoño del iusnaturalismo inmanentista. En esta afirmación se puede compendiar el conjunto de mis hipótesis de trabajo. Esta posibilidad se me presentó como viable durante algún tiempo. En efecto, todo apuntaba a que el aparente dualismo racionalista de la res cogitans y la res extensa habría tenido su continuidad en la escuela wolffiana, por descontado, y más tarde, en la escuela kantiana, bajo idéntico esquema, pero diversa terminología, esta vez separando un uso teorético y un uso práctico de la razón.

Muchos elementos me confirmaban esta hipótesis de trabajo, como se puede observar a lo largo del apartado 2.3 de la investigación: una concepción del ser humano dividido entre el universo de la causalidad mecanicista y el universo del espíritu (apartado 2.3.1), el consiguiente hiato entre la facultad de la razón práctica/voluntad (identificadas) y la facultad del libre arbitrio (apartado 2.3.2), los diversos intentos de establecer distingos entre las expresiones ius naturae, ius naturale, Recht der Natur, natürliches Recht, Naturrecht (apartado 3.1.1), etc. 
Que este esquema dual tenía algo de acertado a la hora de interpretar las fuentes, se me ponía de manifiesto precisamente por el difícil encaje que había de tener el derecho. Ese difícil encaje lo hacía bascular entre la identificación de lo jurídico con lo recto, en último término con la ley (derecho objetivo) y la identificación del derecho con la libertad (derecho subjetivo), según las diversas declaraciones de los autores recogidas en el apartado 3.2.1.1. La pregunta era: el derecho, ¿ética o técnica? A una primera fase de confusión crasa entre derecho y ética, de la mano de Wolff, habría sucedido la celebérrima distinción entre Legalität y Moralität, tantas veces atribuida a Kant, pero que las fuentes demuestran que ya se puede observar en Achenwall, e incluso puede ser rastreada con anterioridad, como se recoge en el apartado 2.3.4 de la investigación. Y así, la progresiva identificación de lo jurídico con el terreno de la mera Legalität, habría otorgado al derecho una naturaleza de tertium genus, a caballo entre la pureza de la moralidad, y el torrente caótico de la empiria.

La continuidad del dualismo racionalista en la escuela kantiana me sirvió de esquema para clasificar la disparidad de criterios utilizados por las fuentes doctrinales en lo referente al objeto formal de la doctrina jurídica, especialmente cuando comenzó a sentirse la necesidad de distinguir entre derecho y ética. Este fue uno de los epígrafes más difíciles de articular, puesto que, como se puede observar a lo largo del apartado 3.2.1, las sucesivas distinciones entre derechos y deberes (Rechte und Pflichten), derechos perfectos e imperfectos (vollkommne und unvollkommne Rechte), derechos interiores y exteriores (innere und äußere Rechte) y derechos coactivos y no coactivos (Zwangsrechte und nicht-Zwangsrechte), no terminaban de dar una respuesta definitiva al estatuto epistémico de la Rechtslehre. Es más, es como si los autores hubieran confiado en que el número de los argumentos aportados pudiera suplir las carencias de cada uno de ellos por separado.

El caso es que, desde aquel dualismo de fondo, adquiría sentido la idea de un distanciamiento entre el plano del derecho natural y el del derecho positivo, y al mismo tiempo, la aparición de una distinción entre un derecho natural puro o absoluto (das reine / absolute Naturrecht) y un derecho natural aplicado o hipotético (das angewandte / hypothetische Naturrecht), según las diversas aproximaciones recogidas en el apartado 3.3. El derecho natural aplicado se 
presentaba como un intento de aterrizar en la realidad concreta las afirmaciones sostenidas a nivel de un derecho natural puro.

En definitiva, la hipótesis de una continuidad entre el vetero y el novoiusnaturalismo, tomada del Prof. Carpintero Benítez (cf. 1989, pp. 16-17), desde la cual el kantismo habría representado el otoño del iusnaturalismo, una prolongación artificiosa de este modo de hacer ciencia jurídica, parecía ponerme sobre la pista correcta: el alejamiento de la realidad y el consiguiente dualismo sistemático, convertía el derecho natural en una entelequia, en las alturas de la pureza racional. Y cada intento para hacer pie en la realidad concreta traía consigo un grado más de creciente exquisitez y filigrana, con sus divisiones y subdivisiones. Es decir, que efectivamente, el novoiusnaturalismo no representaba sino la continuación lógica de la escuela de derecho natural inmanentista, con un refinamiento propio de una etapa de decadencia.

\section{Tesis}

Hasta que, gracias a la intervención de mi Director -el Prof. Palomar Maldonado-, entendí que había llegado el momento de dar el salto desde las hipótesis a la tesis (lo que subyace/lo que se declara), y que expondré sintéticamente a continuación en una sucesión de conclusiones. Lo cual me hizo reflexionar mucho. Porque el término tesis procede del verbo griego $T \imath \theta \dot{\eta} \mu l$, que tiene que ver con poner, lo puesto, o también, lo positivo. Y de pronto, caí en la cuenta de que, a pesar de haberlo leído cientos de veces en las fuentes, había pasado por alto un dato: que el derecho al que invariablemente se referían los autores como derecho tético (positivo) es el derecho natural. En otras palabras, que el derecho natural es el derecho positivo.

Influido por la lectura de las fuentes contemporáneas, y contra la letra de los textos originales, había estado abordando la relación entre derecho natural y derecho positivo como dos órdenes parentéticos, superpuestos. En realidad, y esta es la primera conclusión que destaco, la definición de natura formaliter spectata en Kant es clara (cf. KrV, III B, p.127, Prol., IV, p. 294, KpV, V, p. 43): la existencia de las cosas en cuanto sometidas a leyes (a las leyes del 
entendimiento) o, como se discute en el apartado 2.2.1 de la investigación, la adecuación entre res y lex.

Que la pérdida del ser como acto de los actos del ente había conducido a una metafísica esencialista al wolffianismo era palmario, pero ahora se me abrían nuevas posibilidades interpretativas del sentido de la revolución copernicana desde las que se revelaba la continuidad entre el vetero y el novoiusnaturalismo de la que yo había partido en mis hipótesis, pero a nivel mucho más profundo.

Pues, como señalará el Dr. Petit Sullá (cf. 2004, pp. 81ss.), la suplantación de la física, como estudio de lo natural, por una pretendida metafísica sobre el orden de los conceptos puros del entendimiento, ciertamente buscaba garantizar que este no fuera un orden psicológico, pero tampoco un orden meramente lógico, sino una auténtica lógica trascendental, como analítica de aquella legislación del entendimiento, en cuanto función para todo posible juicio sintético a priori. Y así, entendí la necesidad de una publicación tan tardía como la de la Kritik der Urtheilskraft, en la que se hacía patente la necesidad de atender no a la res, tampoco a la lex, sino a la adecuación entre res y lex, lo cual dejaba al descubierto una realidad: que todo el edificio científico de orientación objetivista de la filosofía criticista descansaba sobre el cimiento inestable del juicio reflexionante, como regla meramente subjetiva, casi una esperanza irracional, en la posibilidad del conocimiento.

Aquella continuidad entre ambas corrientes se hacía más patente cuanto que los presupuestos metafísicos de ambas perdían suelo por ambos extremos: por el lado de la cosa en sí, pero también por el lado de la conciencia del yo, si lo expresamos en términos kantianos. Y eso, aun reconociendo el salto cualitativo que suponía la revolución copernicana respecto de la metafísica de Wolff.

Aquí la atención a la obra del Dr. Canals Vidal fue determinante. Me hizo darme cuenta, y esta es la segunda conclusión que recojo en esta defensa, de que el problema era mucho más profundo de lo que yo había pensado en un principio, pues la hipertrofia de la pregunta por 
los límites del conocer (quomodo se habeat homo ad veritatem cognoscendam), obedecía directamente a la falta de atención por la pregunta sobre el ser del conocer (quod est scire), lo que producía el extraño resultado destacado por Kant en la segunda edición de la Kritik der reinen Vernunft, y decenas de extraños resultados más, en su doctrina y en la de sus discípulos, que anteriormente yo había detectado, pero no sabía cómo integrar.

Porque la unidad de inteligibilidad e intelectualidad en el acto de conocer quedaba subrepticiamente escindida en la equivocidad de la apercepción pura, declarada como Faktum, pero tratada como aquella proposición (yo pienso) en la línea de las verdades objetivas que debe acompañar a todas nuestras representaciones, tal y como se aborda en el apartado 2.2.2.2 de la investigación.

Y entonces entendí el conjunto, tercera conclusión de esta defensa: toda la fuerza argumentativa del iusnaturalismo radicaba en la traspolación analógica de aquella definición de natura formaliter spectata al plano de un uso práctico de la razón, cuyo objeto de estudio había de radicar en la existencia de la cosa, o sea, conciencia empírica en cuanto sometida a la conciencia del yo trascendental, por medio de la legislación moral a priori, en cuanto función para todo juicio moral sintético a priori (juicio de adecuación a la ley o Gesetzmäßigkeit).

La afirmación de Kant en la Kritik der reinen Vernunft, de que a todo juicio analítico lo precede un juicio sintético a priori (cf. III, p. 130) adquiría un sentido más pleno si uno se percata de que, a pesar de los esfuerzos denodados del filósofo de Königsberg para hacer del deber (Sollen) una legislación (Gesetzgebung) de la razón, en realidad, el deber siempre fue abordado no como una legislación, sino como una adecuación a la legislación (Gesetzmäßigkeit), es decir, contrariamente a la letra del propio Kant, como un juicio sintético a priori. Si, ciertamente, la nueva ciencia de la praxis debía venir precedida de una doctrina metafísica de la ética, no es menos cierto que esta debía ser solo una parte de la ciencia práctica en general, la cual requiere a la base un juicio sintético. 
La falta de atención a aquella fundamentación subjetiva que he abordado en el apartado 2.2.2.2, traía como resultado la alineación de la conciencia empírica del individuo con el horizonte del fenómeno, con la consiguiente igualación de todas las operaciones de la voluntad, reducidas a mero arbitrio (Willkühr) (vid. apartado 2.3.2), la degradación de los fines (Zwecke) a simples propósitos (Absicht) (vid. apartado 2.3.5), y la reconfiguración del derecho positivo vigente hasta la fecha, reconociéndolo como mero derecho arbitrario (willkührliches Recht) (vid. apartado 3.1.1).

En ello se puede cifrar la oposición de los kantianos a los desarrollos eudemónicos (basados en la felicidad) y teleiotéticos (basados en la perfección) del veteroiusnaturalismo, pues, siendo así que estos conceptos clave jugaban el mismo papel que el deber en la filosofía criticista, a esta última, sin embargo, se le presentaban como demasiado transidos de fenomenicidad, demasiado dependientes del horizonte de la conciencia empírica.

Así pues, a la analítica de una legislación práctica racional, debía precederle una síntesis a priori, sobre cuya reflexión cobraba sentido el hecho de que fuera adquiriendo una centralidad gradual la distinción entre Legalität y Moralität, tantas veces presentadas como legislación, cuando en realidad son siempre abordadas por los autores como especies del género de la Gesetzmäßigkeit (o sea, la adecuación a la ley).

Síntesis que solo podía dejar de ser problemática en el ser santo, pero que lo era esencialmente respecto de la esencia sensitivo-racional finita, de donde se comprende todo el discurso sobre el carácter coactivo del deber que se recoge en el apartado 2.4, a la hora de abordar la distinción entre la doctrina metafísica del derecho (Rechtslehre) y la doctrina metafísica de la virtud (Tugendlehre).

De esta manera, la apelación a un derecho natural, o una moral natural, casi se tornaba en una petición de principio, la cual había sido resuelta por el veteroiusnaturalismo mediante el recurso a una apelación nominal a la voluntad divina, y más tarde, a medida que fue 
gradualmente vigorizándose el proceso de inmanentización filosófica, mediante el recurso al yo trascendental.

El gran descubrimiento que me permitió poner orden al conjunto, en línea con lo anterior, y que destaco como cuarta conclusión, fue la constatación de que el imperativo categórico, tal y como se suele enunciar, no es un imperativo moral, sino un imperativo jurídico, y se ha de reconocer que los múltiples intentos para enunciarlo de las más diversas maneras no pudieron soslayar jamás esta realidad, ni siquiera los intentos de Feuerbach, o Fichte, que fueron los más audaces en este sentido.

Eso sí, quinta conclusión a destacar: comencé a comprender que el continuismo entre el vetero y el novoiusnaturalismo, no debía impedir reconocer el aporte de Kant, que abordo en el apartado 3.3.1.4, por muy tardía que fuera su célebre Metaphysik der Sitten. Solo asumiendo que el Naturrecht es el auténtico derecho tético, positivo, como se puede observar en el apartado 3.3.2, empecé a percatarme de lo rudimentario que había sido el recurso de wolffianos y kantianos al llamado derecho natural aplicado o hipotético (angewandtes / hypothetisches Naturrecht), como una categoría intermedia entre la doctrina jurídica pura (reines Naturrecht) y el derecho arbitrario.

Aun cuando la fiebre taxonómica fue progresivamente creciente y variable, como se discute a lo largo de los apartados 3.3.3 y 3.3.4, en líneas generales, dentro del angewandtes Naturrecht se entendieron incluidas las grandes instituciones del derecho que siguió llamándose positivo, pero que propiamente deberíamos recibir el nombre de arbitrario: la propiedad (Eigenthum), los contratos (Verträge), los daños (Beleidigungen), y en la mayor parte de los casos, el llamado Gesellschaftsrecht, que yo he preferido traducir como derecho societario.

Sin embargo, pienso firmemente que Kant fue mucho más coherente con los principios del iusnaturalismo cuando en su Metaphysik der Sitten ofreció una clasificación que distinguía llanamente entre derecho privado (Privatrecht) y derecho público (öffentliches Recht) (cf. MS, VI, p. 242). Con esta división, y prescindiendo ya casi por completo del término Naturrecht, 
como se indica al comienzo del apartado 3, se ponía de manifiesto toda la potentísima carga de pretensión histórica universal que, no estando ausente en los demás autores, fue especialmente notable en el filósofo de Königsberg, y que da sentido a la metodología jurídica del iusnaturalismo.

Así, se obtiene que: el derecho natural puro, o derecho tético, es una categoría pura, esta vez no del entendimiento, sino de la razón, que actúa como función para un juicio sintético, por el que el derecho arbitrario o hipotético ha de ser subsumido bajo la premisa primera de la libertad, pero no en un instante y como una mera pretensión teorética, sino a lo largo de un proceso histórico en el que el propio desenvolvimiento del espíritu había de revelar lo arbitrario y parcial (podríamos decir, privado) de todas las manifestaciones morales o jurídicas del estado previo al advenimiento de la comunidad política perfecta, es decir, el llamado Naturstand o estado de naturaleza, entendida esta última en sentido fisicista, como se explicita en el apartado 3.3.2.2.

La pretensión histórica, mucho más presente en la escuela kantiana que en el veteroiusnaturalismo, y mucho más en Kant que en el resto de sus epígonos, centrada, por supuesto, no en la historia que es, sino la historia que debe ser, ofrecía un plan de acción que se desenvuelve en los subepígrafes del apartado 3.3.4, donde al estado de naturaleza (Naturstand), en el que se incluye el derecho societario, le debe suceder el derecho estatal (Staatsrecht), y a este una federación de Estados (der Völkerbund), cuyo derecho de gentes (Völkerrecht) será superado por el derecho del Estado cosmopolita (kosmopolitisches Staatsrecht), derecho plenamente público.

\section{Corolario}

The attempt to set the boundaries of limited knowledge, turned Metaphysics into a formally theoretical but materially practical discipline determining not what knowledge is, but what it ought to be. 
Thus, the research for the rational conditions through which Ethics could and should become science, turned out to be an inquiry for objectivity in a predicamental line. As a consequence, many Wolffian and Kantian scholars dealt with a twofold concept of nature: the horizon of physical phenomena, sheer appearance, and the horizon of spirit. They conceived both as two kinds of legislation.

No wonder what they found objective in the field of theoretical reason, became subjective in the field of a practical use of reason, and vice versa.

'Thou shall not kill', states the commandment. What does it mean? When I am ordered something, for instance, 'not to kill', it is just on the assumption that I am able either to kill or not kill, and I must not kill. What I can do lines up with my status in the world of phenomena, where every action is actually a reaction, i.e. everything is determined by an inexorable chain of causality. Although I have a sphere of action, I should not be properly said to be free. What I can do is not only restricted by what I am forced to do, which was my first hypothesis, but, actually, what I can do is part of what I am forced to do.

A gradual alignment of motivation with the field of phenomenal causality among OldJusnaturalistic scholars was superseded by the Kantian stronger coherence when they identified the empirical consciousness with the rest of phenomena. Empirical consciousness, motivation, subjectivity, prudence, astuteness, technique, etc., these terms were arranged all in a row.

On the other hand, a similar sketch appears beyond the borders of theoretical knowledge: the categorical imperative is not just a limiting commandment, but an enabling precept. What I must do is what I may do, as well.

Why is it that what humankind may do is not what they can do? It is because what empirical individuals have to do (are forced to do) does not coincide with what they must do. Obligations 
have been determined by pathological criteria for centuries. Consequently, it is understandable that Ethics have been mistaken for Morals, that is to say, literally a doctrine about customs. As I stated before, the duality in the concept of nature contains the historical aspiration of Jusnaturalism. The synthetical match between subjective have-to-maxims and the objective must-law is the history of the emancipation of humankind from the chains of causality, and never more appropriately said.

The real enemy for Ethics to show up is nature, when closed in its order, and their subsequent heteronomous Morals. I can conclude: the mix-up over Morals and Law in Old-Jusnaturalism remained in the Kantian mix-up of Ethics and Law. In New-Jusnaturalism there was a splitup between Law and Morals, indeed, yet not a real, but gradual difference between Law and Ethics. Law is the first step to the conformity of the empirical action to the order of freedom. The Metaphysical Doctrine of Law (Rechtslehre) does not expound what law is, but what it ought to be, or what it will be in the future. That is why I purported Metaphysics would become a practical discipline in the Enlightenment Germany. By means of the expansion of war against everyone else, which is Law's goal, virtue will eventually glitter.

In the words of Kant:

„Man kann die Geschichte der Menschengattung im Großen als die Vollziehung eines verborgenen Plans der Natur ansehen, um eine innerlich-, und zu diesem Zwecke, auch äußerlich-vollkommene Staatsverfassung zu Stande zu bringen, als den einzigen Zustand, in welchem sie alle ihre Anlagen in der Menschheit völlig entwickeln kann“" (IGwA, VIII, p. 27).

\section{Conclusiones}

En síntesis, las conclusiones enunciadas ha sido las siguientes:

. Según las fuentes manejadas, el derecho natural es el derecho positivo, puesto por la razón.

Volumen 1. Número 2. Julio - Diciembre 2021 
- La metafísica subyacente al periodo escindió la conciencia en: conciencia empírica y conciencia del yo trascendental, con el consiguiente equivocismo.

. El término naturaleza, como intento de síntesis entre conciencia empírica (res) y conciencia del yo trascendental (lex) tuvo su traslación al plano práctico, de donde la apelación al derecho natural, léase, racional.

. El imperativo categórico no es un imperativo moral, sino jurídico, como función para todo juicio moral en general.

- La síntesis entre conciencia empírica y conciencia del yo trascendental mediante la legislación jurídica/ética, se enuncia como un postulado de tipo histórico, en cuanto tránsito de lo privado a lo público.

Así pues, ciencia, naturaleza e historia son los tres ejes sobre los que bascula toda la investigación.

Mi tesis, por tanto, lo que yo pongo, o propongo, es que, en orden a la comprensión de la ciencia del derecho en Alemania en el periodo que va desde 1750 a 1804, se abandone la hipótesis (su-posición) de que el derecho natural constituye una epéntesis (super-posición) al derecho de la época, y se contemple tal y como lo definen las fuentes, es decir, como derecho tético (positivo). Desdoblaré este corolario de mi tesis en dos apartados: el concepto de derecho, y el concepto de ciencia jurídica.

El término derecho constituyó, tanto para el vetero como para el novoiusnaturalismo, una categoría o concepto puro de la razón, como función necesaria para un juicio sintético (compuesto) a priori, por el que se articularía la adecuación (Gesetzmäßigkeit) de la acción empírica al orden necesario de la libertad. Síntesis (com-posición) problemática, dada la heterogeneidad (antítesis, contra-posición) entre el orden físico de la naturaleza y el orden hiperfísico de la libertad. 
El reconocimiento del carácter esencialmente coactivo y al mismo tiempo natural del derecho, no fue una contradicción, por cuanto a las fuentes tanto del vetero como del novoiusnaturalismo siempre les estuvo presente que no es la naturaleza la que contiene a la libertad, sino la libertad la que contiene a la naturaleza, de donde se comprende la afirmación del primado de la razón práctica por parte de Kant (vid. apartado 2.1). Pues el orden jurídico había de ser coactivo para el orden de la naturaleza, pero no para el orden del espíritu, solo respecto del cual el derecho tético podía ser llamado natural. Se ha de recordar que el orden jurídico no es el orden de la mera coacción, sino el orden de la coacción legítima, válida, es decir, adecuada al orden racional, de modo que el orden de la naturaleza sea modelado, en un proceso de tipo histórico, a imagen y semejanza el orden del espíritu.

Es por esto que cabe concluir que la ciencia jurídica (Rechtslehre) es definida, de acuerdo con las fuentes del periodo, como aquel sistema racional autorreferencial destinado a legitimar la acción manipuladora del espíritu sobre la naturaleza, con los instrumentos causales propios de esta última (la coacción), al objeto de privar a la esencia racional finita de su ecología propia, y servir como motor de cambio histórico, de modo que la conciencia empírica se adecúe y subsuma al orden de la libertad, en un primer momento de un modo exterior, es decir, por cualesquiera motivos (Legalität), pero ello como una propedéutica para un mundo futuro en el que la síntesis entre conciencia empírica y conciencia objetiva del yo trascendental llegue al grado de suprema perfección e identificación (Moralität).

En definitiva, como recojo en las conclusiones: de acuerdo con las fuentes, el fin del derecho, según su desenvolvimiento histórico, consiste en vencer la resistencia del principio malo inserto en el corazón del hombre, de modo que su facultad de desear, purificada de elementos patológicos, se adecúe interiormente a lo absoluto indeterminado de la libertad.

La ciencia jurídica, atendidas las fuentes, constituiría la exposición sistemática del derecho como un artefacto provisional de la razón, que ha de posibilitar un tránsito en el que la humanidad de la esencia racional finita llegue a identificarse, en el punto omega de la historia, con el ser santo, de modo que su buena voluntad, adecuada apriorísticamente con el imperativo 
categórico, alumbre un mundo nuevo en el que el género humano, emancipado de las cadenas de la naturaleza física, alcance el supremo bien, que consiste en la felicidad sin límites, habiéndola merecido. Y esto es lo que Kant llama el Quiliasmo de la filosofía (IGwA, VIII, p. 27). Genug.

\section{Referencias}

Blüdhorn, J. (1973). „Kantianer“ und Kant. Die Wende von der Rechtsmetaphysik zur „Wissenschaft“ vom positiven Recht. Kant-Studien, 64(1), pp. 363-394.

Canals Vidal, F. (2013-19). Obras Completas (Vol. II, IVB, VI). Barcelona: Balmes.

Canals Vidal, F. (1987) Sobre la esencia del conocimiento. Barcelona: Promociones Publicaciones Universitarias.

Carpintero Benítez, F. (1988). Una introducción a la ciencia jurídica. Madrid: Civitas.

Carpintero Benítez, F. (1989). La cabeza de Jano. Cádiz: Servicio de Publicaciones de la Universidad de Cádiz.

Klippel, D. (1976). Politische Freiheit und Freiheitsrechte im deutschen Naturrecht des 18. Jahrhunderts. Padenborn: Ferdinand Schöningh.

Königlich Preußische Akademie der Wissenschaften (ed.) (1900ss.). Kants gesammelte Schriften (XXIV Bände). Berlín: Georg Reimer / de Gruyter (desde 1922). Para las citas a cuerpo de texto, se indican las apócopes más habituales de las obras de Kant, el volumen al que corresponden, y la página concreta. En lo referente a la Kritik de reinen Vernunft, la letra B hace referencia a la segunda edición de esta obra.

Liebmann, O. (1865). Kant und die Epigonen. Stuttgart: Carl Schober. 
Petit Sullá, J.M. (2011), Obras Completas. Madrid: Edición de José María Alsina Roca y Evaristo Palomar Maldonado.

Petit Sullá, J.M. \& Prevosti Monclús, A. (2004) Filosofía de la naturaleza. Barcelona: Scire.

Schröder, J. \& Pielemeier, I. (1995), „Naturrecht als Lehrfach an den deutschen Universitäten des 18. und 19. Jahrhunderts“. En Dann, O. \& Klippel, D. (ed.) Naturrecht - Spätaufklärung - Revolution (pp. 255-269). Hamburg: Felix Meiner Verlag.

Warnkönig, L.A. (1839). Rechtsphilosophie als Naturlehre des Rechts. Freiburg im Breisgau: Fr. Wagnerschen Buchhandlung. 
REVISTA INTERNACIONAL DE FILOSOFÍA TEÓRICA Y PRÁCTICA

Volumen 1. Número 2. Julio - Diciembre 2021 ISSN: 2745-0333 (En línea) 